\title{
GEOMETRIC CHARACTERIZATION OF HERMITIAN ALGEBRAS WITH CONTINUOUS INVERSION
}

\author{
DANIEL BELTIŢĂ ${ }^{凶}$ and KARL-HERMANN NEEB
}

(Received 11 March 2009)

\begin{abstract}
A hermitian algebra is a unital associative $\mathbb{C}$-algebra endowed with an involution such that the spectra of self-adjoint elements are contained in $\mathbb{R}$. In the case of an algebra $\mathcal{A}$ endowed with a Mackey-complete, locally convex topology such that the set of invertible elements is open and the inversion mapping is continuous, we construct the smooth structures on the appropriate versions of flag manifolds. Then we prove that if such a locally convex algebra $\mathcal{A}$ is endowed with a continuous involution, then it is a hermitian algebra if and only if the natural action of all unitary groups $\mathrm{U}_{n}(\mathcal{A})$ on each flag manifold is transitive.
\end{abstract}

2000 Mathematics subject classification: primary 46K05; secondary 22E65, 58B10, 46H30.

Keywords and phrases: algebra with continuous inversion, flag manifold, locally convex Lie group.

\section{Introduction}

There exists a deep relationship between the locally convex topological algebras with continuous inversion and Lie theory. This fundamental knowledge originated in the paper [G102] and has been crucial for many of the subsequent advances in the theory of infinite-dimensional Lie groups modeled on locally convex spaces; see for instance the survey [Ne06] and the forthcoming monograph [GIN09].

On the other hand, a large part of the earlier research in this area focused on Banach manifolds and their symmetry groups. This was naturally related to the spectral theory of Hilbert space operators and has led to many deep results. A good source of information about this connection is the monograph [Up85].

It is one of the aims of the present paper to relate the themes mentioned above, by pointing out that the spectral properties in involutive topological algebras are intimately connected with differential geometric properties of certain Lie groups and homogeneous spaces associated with the algebras under consideration. Specifically, we prove that a Mackey-complete algebra $\mathcal{A}$ with continuous inversion is hermitian (that is, the spectrum of every self-adjoint element is contained in $\mathbb{R}$ ) if and only if the unitary group of the matrix algebra $M_{2}(\mathcal{A})$ acts transitively on the corresponding flag

(C) 2009 Australian Mathematical Publishing Association Inc. 0004-9727/2009 \$16.00 
manifolds (Corollary 4.7 below). This result seems to be new even in the special case when $\mathcal{A}$ is a Banach algebra. However, the Banach setting is not wide enough to cover some of the most important situations, particularly those coming from the theory of loop groups and gauge theory; see Example 2.7 for further details.

The aforementioned flag manifolds are well-known generalizations of the projective spaces and were traditionally studied in complex and algebraic geometry. There exist a number of infinite-dimensional versions of these compact complex manifolds, which are homogeneous Banach manifolds and play an interesting role in operator theory (see, for instance, [Up85] or [B106]) or in the theory of loop groups and certain areas of mathematical physics [PS90]. We recall that the construction of smooth structures on homogeneous spaces of infinite-dimensional Lie groups is often a difficult issue since it usually relies on the inverse mapping theorem, which fails beyond the setting of Banach spaces; even in this setting, one additionally needs to find complements for subspaces in Banach spaces, which is often a rather difficult task. From this point of view, a by-product of the present research turns out to be particularly important: we show that by just using an appropriate Gauß decomposition for matrices with entries in a topological algebra, it is possible to construct adequate smooth structures on the flag manifolds associated with the continuous inverse algebras (Theorem 4.3). The core of our method is a very general lemma that is interesting on its own, which we have recorded in Appendix A.

The methods used in this paper are similar to those used in [BrN05] in a very general context to obtain manifold structures on homogeneous spaces associated to 3-graded Lie algebras, which leads in particular to natural manifold structures on the generalized Graßmannians, obtained as orbits of projections. We plan to use the results of the present paper, such as Corollary 3.7, to study representations in spaces of sections of a holomorphic vector over these manifolds (see [MNS09] for some results in the Banach context).

\section{Definitions and examples}

Notation 2.1. For an arbitrary unital complex associative algebra $\mathcal{A}$ we use the following notation:

- $\mathcal{A}^{\times}=\left\{a \in \mathcal{A} \mid\left(\exists a^{-1} \in \mathcal{A}\right) \quad a a^{-1}=a^{-1} a=\mathbf{1}\right\}$;

- $\quad$ the spectrum of any $a \in \mathcal{A}$ is $\sigma_{\mathcal{A}}(a)=\sigma(a)=\left\{\lambda \in \mathbb{C} \mid \lambda \mathbf{1}-a \notin \mathcal{A}^{\times}\right\}$.

In addition, if $\mathcal{A}$ is endowed with an involution $a \mapsto a^{*}$, the we use the notation:

- $\quad$ the unitary group $\mathrm{U}(\mathcal{A})=\left\{u \in \mathcal{A}^{\times} \mid u^{*} u=\mathbf{1}\right\}$;

- $\quad$ the set of nonnegative elements $\mathcal{A}_{+}=\left\{a \in \mathcal{A} \mid a=a^{*}\right.$ and $\left.\sigma_{\mathcal{A}}(a) \subseteq[0, \infty)\right\}$;

- $\quad$ the set of positive elements $\mathcal{A}_{+}^{\times}=\mathcal{A}^{\times} \cap \mathcal{A}_{+}$.

Moreover, for any complex vector space $\mathcal{X}$ we denote by $\mathcal{L}(\mathcal{X})$ the set of all linear maps from $\mathcal{X}$ into itself.

Definition 2.2. Let $\mathcal{A}$ be an associative unital complex algebra endowed with an involution $a \mapsto a^{*}$. We say that $\mathcal{A}$ is a hermitian algebra if $\sigma_{\mathcal{A}}(a) \subseteq \mathbb{R}$ whenever $a=a^{*} \in \mathcal{A}$. 
For use later we record the following sufficient condition for an involutive algebra to be hermitian.

REMARK 2.3. If $\mathcal{A}$ is an associative unital complex algebra endowed with an involution and for every $a=a^{*} \in \mathcal{A}$ we have $-1 \notin \sigma_{\mathcal{A}}\left(a^{2}\right)$, then $\mathcal{A}$ is a hermitian algebra.

In order to prove this assertion, let us first note that for every $a \in \mathcal{A}$ we have $\sigma_{\mathcal{A}}\left(a^{2}\right) \supseteq\left\{\lambda^{2} \mid \lambda \in \sigma_{\mathcal{A}}(a)\right\}$. In fact, if $\lambda \in \mathbb{C}$ and $\lambda^{2} \in \mathbb{C} \backslash \sigma_{\mathcal{A}}\left(a^{2}\right)$, then there exists $b \in \mathcal{A}$ with $b\left(\lambda^{2} \mathbf{1}-a^{2}\right)=\left(\lambda^{2} \mathbf{1}-a^{2}\right) b=\mathbf{1}$. Then $\lambda \mathbf{1}-a$ has both a left inverse and a right inverse, hence it belongs to $\mathcal{A}^{\times}$, and then $\lambda \in \mathbb{C} \backslash \sigma_{\mathcal{A}}(a)$.

Now let $a=a^{*} \in \mathcal{A}$ and assume that $\sigma_{\mathcal{A}}(a) \nsubseteq \mathbb{R}$, so there exists $\lambda \in \sigma_{\mathcal{A}}(a) \backslash \mathbb{R}$. Then $\lambda=x+\mathrm{i} y$ with $x, y \in \mathbb{R}$ and $y \neq 0$, whence $\mathrm{i} \in \sigma((1 / y)(a-x \mathbf{1}))$. Thence $-1=\mathrm{i}^{2} \in \sigma\left(((1 / y)(a-x \mathbf{1}))^{2}\right)$ by the above remark, and this contradicts the assumption on $\mathcal{A}$ since $(1 / y)(a-x \mathbf{1})$ is a self-adjoint element in $\mathcal{A}$.

DEFINITION 2.4. A continuous inverse algebra (CIA) is a Hausdorff locally convex unital algebra $\mathcal{A}$ whose unit group $\mathcal{A}^{\times}$is open and for which the inversion map $\mathcal{A}^{\times} \rightarrow \mathcal{A}, a \mapsto a^{-1}$ is continuous.

If, in addition, $\mathcal{A}$ is complete, then the same arguments as for Banach algebras lead to a holomorphic functional calculus [G102, Wa67]. Since completeness is in general not inherited by quotients [Ko69, Section 31.6], it is natural to consider for CIAs the weaker condition that they are FC-complete in the sense that they are closed under holomorphic functional calculus (see [B1N08]). This means that for $a \in A$, any open neighborhood $U$ of $\sigma(a)$, each holomorphic function $f \in \mathcal{O}(U)$ and any contour $\Gamma$ around $\sigma(a)$ in $U$, the integral

$$
f(a):=\frac{1}{2 \pi i} \oint_{\Gamma} f(\zeta)(a-\zeta \mathbf{1})^{-1} d \zeta,
$$

which defines an element of the completion of $\mathcal{A}$, actually exists in $\mathcal{A}$.

REMARK 2.5. A discussion of hermitian algebras with continuous inversion including various equivalent characterizations in the case of Mackey complete algebras can be found in [Bi09, Section 7].

It is well known that the $C^{*}$-algebras are hermitian algebras. Here are two important examples that go beyond the traditional setting of operator algebras.

EXAMPLE 2.6 (Group algebras). Let $G$ be any finite-dimensional connected nilpotent Lie group. Then the unitization of the group algebra $L^{1}(G)$ is always a hermitian Banach algebra; see [Po77]. See [FGL06, Le76, Ku79] for a discussion of more general versions of group algebras that give rise to hermitian Banach algebras.

EXAMPLE 2.7 (Loop algebras). Let $\mathbb{T}$ denote the one-dimensional torus and $\mathcal{A}:=$ $\mathcal{C}^{\infty}\left(\mathbb{T}, M_{n}(\mathbb{C})\right)$ for some $n \geq 1$. Then $\mathcal{A}$ endowed with the pointwise defined operations has a natural structure of a hermitian (non-Banach) algebra with continuous inversion, which plays a central role in the theory of loop groups, in as much as $\mathcal{A}^{\times}$ 
is precisely the loop group associated with the general linear group $\mathrm{GL}_{n}(\mathbb{C})$ (see, for instance, [PS90]).

More generally, one can prove that if $\mathcal{A}$ is a hermitian algebra with continuous inversion and $M$ is a compact topological space, then the algebra $\mathcal{C}(M, \mathcal{A})$ of continuous $\mathcal{A}$-valued functions on $M$ with the pointwise defined operations is in turn a hermitian algebra with continuous inversion. A similar assertion holds for the algebra $\mathcal{C}^{\infty}(M, \mathcal{A})$ of smooth $\mathcal{A}$-valued functions on $M$ provided that $M$ is a compact manifold; see [G102] and [Ne06, Examples VIII.3].

Definition 2.8. For any unital involutive complex algebra $\mathcal{A}$ we denote $\mathcal{P}(\mathcal{A})=$ $\left\{p \in \mathcal{A} \mid p=p^{*}=p^{2}\right\}$. For $p, q \in \mathcal{A}$ the notation $p \leq q$ means that $q p=p$. If, in addition, $p \neq q$, then we write $p<q$.

Now assume that $\delta$ : $0=p_{0}<p_{1}<\cdots<p_{n}=\mathbf{1}$ is a finite, totally ordered family of elements in $\mathcal{P}_{\mathcal{A}}$. We define the mapping of diagonal truncation

$$
\Phi_{\delta}: \mathcal{A} \rightarrow \mathcal{A}, \quad x \mapsto \sum_{k=1}^{n}\left(p_{k}-p_{k-1}\right) x\left(p_{k}-p_{k-1}\right)
$$

and the unital associative subalgebra of $\mathcal{A}$,

$$
\begin{aligned}
\Delta(\delta) & :=\left\{x \in \mathcal{A} \mid x p_{k}=p_{k} x p_{k} \text { for } k=0,1, \ldots, n\right\} \\
& =\left\{x \in \mathcal{A} \mid x p_{k} \mathcal{A} \subseteq p_{k} \mathcal{A} \text { for } k=0,1, \ldots, n\right\},
\end{aligned}
$$

which is the stabilizer of the flag $\left(p_{0} \mathcal{A}, \ldots, p_{n} \mathcal{A}\right)$ of right ideals. Note that the restriction of $\Phi_{\delta}$ to the algebra $\Delta(\delta)$ is multiplicative. Also, $\Phi_{\delta}$ is an idempotent mapping and its range is a unital $*$-subalgebra of $\mathcal{A}$ which can be described as

$$
D(\delta):=\operatorname{Ran}\left(\Phi_{\delta}\right)=\left\{x \in \mathcal{A} \mid x p_{k}=p_{k} x \text { for } k=0,1, \ldots, n\right\} .
$$

We also denote $N(\delta):=\Delta(\delta) \cap\left(\Phi_{\delta}\right)^{-1}(\mathbf{1})$, which is a group of invertible elements in $\Delta(\delta)$.

EXAMPLE 2.9. Let $\mathcal{B}$ be a unital involutive complex algebra and $n \geq 1$ arbitrary. Then the matrix algebra $\mathcal{A}:=M_{n}(\mathcal{B}):=M_{n}(\mathbb{C}) \otimes \mathcal{B}$ has a natural structure of unital involutive complex algebra and if we define $p_{1}, p_{2}, \ldots, p_{n} \in \mathcal{A}$ by

$$
p_{1}=\left(\begin{array}{ccccc}
1 & 0 & 0 & \ldots & 0 \\
0 & 0 & 0 & \ldots & 0 \\
0 & 0 & 0 & \ldots & 0 \\
\vdots & \vdots & \vdots & \ddots & \vdots \\
0 & 0 & 0 & \ldots & \vdots
\end{array}\right), \quad p_{2}=\left(\begin{array}{ccccc}
1 & 0 & 0 & \ldots & 0 \\
0 & 1 & 0 & \ldots & 0 \\
0 & 0 & 0 & \ldots & 0 \\
\vdots & \vdots & \vdots & \ddots & \vdots \\
0 & 0 & 0 & \ldots & 0
\end{array}\right), \ldots, \quad p_{n}=\left(\begin{array}{ccccc}
1 & 0 & 0 & \ldots & 0 \\
0 & 1 & 0 & \ldots & 0 \\
0 & 0 & 1 & \ldots & 0 \\
\vdots & \vdots & \vdots & \ddots & \vdots \\
0 & 0 & 0 & \ldots & \mathbf{1}
\end{array}\right),
$$

then we obtain a totally ordered family $\delta$ : $0=p_{0}<p_{1}<\cdots<p_{n}=\mathbf{1}$. The corresponding mapping $\Phi_{\delta}: \mathcal{A} \rightarrow \mathcal{A}$ is defined by replacing the off-diagonal entries 
by zeros,

$$
\left(\begin{array}{ccccc}
b_{11} & b_{12} & b_{13} & \ldots & b_{1 n} \\
b_{21} & b_{22} & b_{23} & \ldots & b_{2 n} \\
b_{31} & b_{32} & b_{33} & \ldots & b_{3 n} \\
\vdots & \vdots & \vdots & \ddots & \vdots \\
b_{n 1} & b_{n 2} & 0 & \ldots & b_{n n}
\end{array}\right) \longmapsto\left(\begin{array}{ccccc}
b_{11} & 0 & 0 & \ldots & 0 \\
0 & b_{22} & 0 & \ldots & 0 \\
0 & 0 & b_{33} & \ldots & 0 \\
\vdots & \vdots & \vdots & \ddots & \vdots \\
0 & 0 & 0 & \ldots & b_{n n}
\end{array}\right)
$$

while $\Delta(\delta)$ is the algebra of upper triangular matrices in $\mathcal{A}=M_{n}(\mathcal{B})$.

\section{Factorizations}

In this section we provide an extension of [Pi88, Proposition 3.1] from the setting of von Neumann algebras to that of hermitian algebras with continuous inversion; see Proposition 3.6 below. This will be a key tool in our geometric characterization of hermitian algebras in terms of flag manifolds (Theorem 4.5 and Corollary 4.7).

Lemma 3.1. Let $\mathcal{A}$ be a unital associative algebra and $p \in \mathcal{P}(\mathcal{A})$. Consider the unital algebra $\mathcal{A}_{p}:=p \mathcal{A} p$ with the unit $p$, and define $\iota_{0}: \mathcal{A}_{p} \hookrightarrow \mathcal{A}$ (the inclusion map) and $\iota_{1}: \mathcal{A}_{p} \rightarrow \mathcal{A}, x \mapsto x+(1-p)$, which is an inclusion of multiplicative monoids. If $p \neq \mathbf{1}$, then for every $x \in \mathcal{A}_{p}$ we have $\sigma_{\mathcal{A}}\left(\iota_{0}(x)\right)=\sigma_{\mathcal{A}_{p}}(x) \cup\{0\}$ and $\sigma_{\mathcal{A}}\left(\iota_{1}(x)\right)=\sigma_{\mathcal{A}_{p}}(x) \cup\{1\}$.

PROOF. The first of these equalities is equivalent to $\mathbb{C}^{\times} \backslash \sigma_{\mathcal{A}_{p}}(x)=\mathbb{C} \backslash \sigma_{\mathcal{A}}\left(\iota_{0}(x)\right)$. To prove the inclusion $\subseteq$, let $\lambda \in \mathbb{C}^{\times} \backslash \sigma_{\mathcal{A}_{p}}(x)$ be arbitrary. Then there exists $b \in \mathcal{A}_{p}$ such that $(\lambda p-x) b=b(\lambda p-x)=p$. Since $b p=p b$, it then follows that $(\lambda \mathbf{1}-x)\left(b+\lambda^{-1}(\mathbf{1}-p)\right)=((\lambda p-x)+\lambda(\mathbf{1}-p))\left(b+\lambda^{-1}(\mathbf{1}-p)\right)=$ $p+(\mathbf{1}-p)=\mathbf{1}$, and similarly $\left(b+\lambda^{-1}(\mathbf{1}-p)\right)(\lambda \mathbf{1}-x)=\mathbf{1}$. Thus, $\lambda \mathbf{1}-x$ has the inverse $b+\lambda^{-1}(\mathbf{1}-p) \in \mathcal{A}$. Since $\iota_{0}(x)=x$, in particular we get $\lambda \in \mathbb{C} \backslash \sigma_{\mathcal{A}}\left(\iota_{0}(x)\right)$. Conversely, assume the latter condition. Then there exists $c \in \mathcal{A}$ such that $(\lambda \mathbf{1}-x)$ $c=c(\lambda \mathbf{1}-x)=\mathbf{1}$. As $p x=x p$, it follows at once that $p c=c p \in \mathcal{A}_{p}$, and then $(\lambda p-x) p c=p(\lambda \mathbf{1}-x) c=p$ and $p c(\lambda p-x)=c(\lambda \mathbf{1}-x) p=p$. Thus, $\lambda p-x$ has the inverse $p c \in \mathcal{A}_{p}$, and in particular $\lambda \in \mathbb{C} \backslash \sigma_{\mathcal{A}_{p}}(x)$. If $\lambda=0$, then $x c=c x=\mathbf{- 1}$ hence $x$ is invertible in $\mathcal{A}$; on the other hand, since $x \in \mathcal{A}_{p}$, we have $x(\mathbf{1}-p)=0$, and then the fact that $x$ is invertible implies $\mathbf{1}-p=0$, which contradicts our hypothesis. Thus, $\lambda \neq 0$, and then $\lambda \in \mathbb{C}^{\times} \backslash \sigma_{\mathcal{A}_{p}}(x)$ as required.

The proof of the second of the asserted equalities relies on a similar method. We actually check that $(\mathbb{C} \backslash\{1\}) \backslash \sigma_{\mathcal{A}_{p}}(x)=\mathbb{C} \backslash \sigma_{\mathcal{A}_{p}}\left(\iota_{1}(x)\right)$. If $\lambda$ is an arbitrary element in the left-hand side of this equation and $b \in \mathcal{A}_{p}$ is the inverse of $\lambda p-x$, then $b+(\lambda-1)^{-1}(\mathbf{1}-p)$ turns out to be the inverse of $\lambda \mathbf{1}-\iota_{1}(x)$. Conversely, if $\lambda \in \mathbb{C} \backslash \sigma_{\mathcal{A}_{p}}\left(\iota_{1}(x)\right)$ and $d$ is the inverse of $\lambda \mathbf{1}-\iota_{1}(x)$ in $\mathcal{A}$, then $p d=d p \in \mathcal{A}_{p}$ and this element is the inverse of $\lambda p-x$ in $\mathcal{A}_{p}$.

The first part of the following statement was also noted in [DG01, Remark 7.1]; see [Ne08, Lemma 1.2]. 
Proposition 3.2. Let $\mathcal{A}$ be a unital algebra with continuous inversion and $p \in$ $\mathcal{P}(\mathcal{A})$. Then the unital algebra $\mathcal{A}_{p}:=p \mathcal{A} p$ with the unit $p$ and endowed with the relative topology is in turn an algebra with continuous inversion. In addition, if $\mathcal{A}$ is a hermitian algebra with continuous inversion and $p=p^{*}$, then $\mathcal{A}_{p}$ is in turn a hermitian algebra with respect to the involution induced from $\mathcal{A}$ and

$$
\left(\mathcal{A}_{p}\right)^{\times}=\iota_{1}^{-1}\left(\mathcal{A}^{\times}\right),
$$

where $\iota_{1}: \mathcal{A}_{p} \rightarrow \mathcal{A}, x \mapsto x+\mathbf{1}-p$.

PROOF. It is clear that the continuous map $\iota_{1}: \mathcal{A}_{p} \rightarrow \mathcal{A}$, is multiplicative, satisfies $\left(\mathcal{A}_{p}\right)^{\times}=\iota^{-1}\left(\mathcal{A}^{\times}\right)$, and intertwines the inversion mappings on $\left(\mathcal{A}_{p}\right)^{\times}$and $\mathcal{A}^{\times}$. This shows that $\left(\mathcal{A}_{p}\right)^{\times}$is an open subset of $\mathcal{A}_{p}$ and the inversion mapping is continuous on $\left(\mathcal{A}_{p}\right)^{\times}$.

Now assume that $\mathcal{A}_{p}$ is a hermitian algebra and let $x=x^{*} \in \mathcal{A}_{p}$ be arbitrary. It then follows by Lemma 3.1 that $\sigma_{\mathcal{A}_{p}}(x) \subseteq \sigma_{\mathcal{A}}(x) \subseteq \mathbb{R}$, which concludes the proof.

In the special case when $\mathcal{A}$ is a Banach algebra, the conclusion of the following proposition is well known; see, for instance, [Le76, Assertion (4)]. In order to obtain this result in the general situation, we rely on a purely algebraic result established in [Wi76].

Proposition 3.3. Let $\mathcal{A}$ be a CIA endowed with a continuous involution $a \mapsto a^{*}$. If $\mathcal{A}$ is Mackey complete, then for every $n \geq 1$ the following assertions are equivalent:

(1) the algebra $\mathcal{A}$ is hermitian;

(2) the matrix algebra $M_{n}(\mathcal{A})$ is hermitian.

PROOF. First recall that the matrix algebra $M_{n}(\mathcal{A})$ is in turn a CIA (see [Sw77, Corollary 1.2]). The implication (2) implies (1) follows by applying Proposition 3.2 for the self-adjoint idempotent element $p=p_{1}$ of Example 2.9.

Conversely, assume that $\mathcal{A}$ is a hermitian algebra. Then, according to the ShiraliFord theorem for algebras with continuous inversion, $\mathcal{A}$ has the property that every element of the form $a_{1}^{*} a_{1}+\cdots+a_{k}^{*} a_{k}$ has the spectrum contained in $[0, \infty)$, for arbitrary $k \geq 1$ and $a_{1}, \ldots, a_{k} \in \mathcal{A}$ (see [Bi09, Proposition 6.8 and Corollary 7.7]). Then the theorem proved in [Wi76] shows that the matrix algebra $M_{n}(\mathcal{A})$ has a similar property. In particular, for every matrix $a \in M_{n}(\mathcal{A})$, the spectrum of $a^{*} a \in M_{n}(\mathcal{A})$ is contained in $[0, \infty)$. Then it follows by the above Remark 2.3 that $M_{n}(\mathcal{A})$ is a hermitian algebra.

Proposition 3.4. Let $\mathcal{A}$ be a Mackey-complete, hermitian $C I A, p=p^{*} \in \mathcal{P}(\mathcal{A})$, and $\mathcal{A}_{p}=p \mathcal{A} p$. Then for every $a \in \mathcal{A}^{\times}$we have pa* ap $\in\left(\mathcal{A}_{p}\right)^{\times}$.

PROOF. It follows by Proposition 3.2 that $\mathcal{A}_{p}$ is a hermitian CIA. On the other hand, $\mathcal{A}_{p}$ is clearly Mackey-complete because $\mathcal{A}$ is. Now for $\mathcal{B}=\mathcal{A}$ or $\mathcal{B}=\mathcal{A}_{p}$ define the function

$$
\tau_{\mathcal{B}}: \mathcal{B} \rightarrow[0, \infty), \quad \tau_{\mathcal{B}}(a)=\left(r_{\mathcal{B}}\left(a^{*} a\right)\right)^{1 / 2} .
$$


As a consequence of Lemma 3.1, the functions $\tau_{\mathcal{A}_{p}}$ and $\tau_{\mathcal{A}}$ agree, in the sense that $\tau_{\mathcal{A}_{p}}=\left.\tau_{\mathcal{A}}\right|_{\mathcal{A}_{p}}$. It then follows by the condition '(i) if and only if (iii)' in [Bi09, Theorem 7.3] that the enveloping $C^{*}$-algebra $C^{*}\left(\mathcal{A}_{p}\right)$ is a $C^{*}$-subalgebra of $C^{*}(\mathcal{A})$ and the diagram

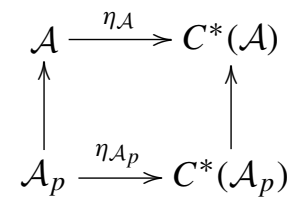

is commutative, where the vertical arrows are inclusion maps and $\eta_{\mathcal{B}}: \mathcal{B} \rightarrow C^{*}(\mathcal{B})$ stands for the canonical $*$-morphism for $\mathcal{B}=\mathcal{A}$ or $\mathcal{B}=\mathcal{A}_{p}$ (see [Bi09, Definition 3.7]).

Note that $C^{*}\left(\mathcal{A}_{p}\right)$ may not contain the unit element $\mathbf{1}=\eta_{\mathcal{A}}(\mathbf{1}) \in C^{*}(\mathcal{A})$, however it is a unital $C^{*}$-algebra in its own right, with the unit element $\eta_{\mathcal{A}}(p)\left(=\eta_{\mathcal{A}_{p}}(p)\right)$. We are going to check that actually

$$
C^{*}\left(\mathcal{A}_{p}\right)=C^{*}(\mathcal{A})_{\eta_{\mathcal{A}}(p)}:=\eta_{\mathcal{A}}(p) \cdot C^{*}(\mathcal{A}) \cdot \eta_{\mathcal{A}}(p) .
$$

In fact, since $p=p^{*}=p^{2}$ it follows that $\eta_{\mathcal{A}}(p)=\eta_{\mathcal{A}}(p)^{*}=\eta_{\mathcal{A}}(p)^{2}$, whence

$$
C^{*}(\mathcal{A})_{\eta_{\mathcal{A}}(p)}=\left\{b \in C^{*}(\mathcal{A}) \mid b \eta_{\mathcal{A}}(p)=\eta_{\mathcal{A}}(p) b\right\} .
$$

Since $p$ is the unit element of $\mathcal{A}_{p}$, it follows that $\eta_{\mathcal{A}_{p}}\left(\mathcal{A}_{p}\right) \subseteq C^{*}(\mathcal{A})_{\eta_{\mathcal{A}}(p)}$, so $C^{*}\left(\mathcal{A}_{p}\right) \subseteq C^{*}(\mathcal{A})_{\eta_{\mathcal{A}}(p)}$, since the range of $\eta_{\mathcal{A}_{p}}$ is dense in $C^{*}\left(\mathcal{A}_{p}\right)$. Conversely, let $b \in C^{*}(\mathcal{A})_{\eta_{\mathcal{A}}(p)}$ arbitrary. In particular, $b \in C^{*}(\mathcal{A})$ hence there exists a net $\left\{a_{j}\right\}_{j \in J}$ such that $b=\lim _{j \in J} \eta_{\mathcal{A}}\left(a_{j}\right)$. On the other hand, since $b \in C^{*}(\mathcal{A})_{\eta_{\mathcal{A}}(p)}$ we obtain $b=\eta_{\mathcal{A}}(p) b \eta_{\mathcal{A}}(p)=\eta_{\mathcal{A}}(p) \lim _{j \in J} \eta_{\mathcal{A}}\left(a_{j}\right) \eta_{\mathcal{A}}(p)=\lim _{j \in J} \eta_{\mathcal{A}}\left(p a_{j} p\right) \in$ $C^{*}\left(\mathcal{A}_{p}\right)$, and (3.2) is proved.

We now come back to the proof of the assertion. For $a \in \mathcal{A}^{\times}$it follows by [Bi09, Proposition 7.5] that $\eta_{\mathcal{A}}(a) \in C^{*}(\mathcal{A})^{\times}$, whence $\eta_{\mathcal{A}}(p) \eta_{\mathcal{A}}(a)^{*} \eta_{\mathcal{A}}(a) \eta_{\mathcal{A}}(p) \in$ $\left(C^{*}(\mathcal{A})_{\eta_{\mathcal{A}}(p)}\right)^{\times}$. (The latter fact follows for instance by considering a faithful representation of $C^{*}(\mathcal{A})$ on some Hilbert space and using the fact that a positive operator on a Hilbert space is invertible if and only if it is bounded from below by some positive scalar multiple of the identity.) Then by (3.2) and (3.1) we obtain $\eta_{\mathcal{A}_{p}}\left(p a^{*} a p\right) \in C^{*}\left(\mathcal{A}_{p}\right)^{\times}$, and now by [Bi09, Proposition 7.5] again it follows that $p a^{*} a p \in\left(\mathcal{A}_{p}\right)^{\times}$.

Corollary 3.5. Let $\mathcal{A}$ be a Mackey-complete, hermitian algebra with continuous inversion. Assume that $p=p^{*} \in \mathcal{P}(\mathcal{A})$ and denote $\mathcal{A}_{p}=p \mathcal{A} p$. Then for every $a \in \mathcal{A}^{\times}$there exists $b \in\left(\mathcal{A}_{p}\right)^{\times}$such that $p a^{*} a p=b^{*} b$. In addition, the invertible element $b$ can be chosen such that $b=b^{*}$ and $\sigma_{\mathcal{A}}(b) \subseteq(0, \infty)$.

PROOF. It follows by Proposition 3.2 that $\mathcal{A}_{p}$ is a hermitian algebra with continuous inversion, and then by [Bi09, Proposition 7.5] we obtain $\sigma_{\mathcal{A}_{p}}\left(p a^{*} a p\right)=$ $\sigma_{C^{*}\left(\mathcal{A}_{p}\right)}\left(\eta_{\mathcal{A}_{p}}\left(p a^{*} a p\right)\right) \subseteq[0, \infty)$. On the other hand $p a^{*} a p \in\left(\mathcal{A}_{p}\right)^{\times}$by Proposition 3.4, hence actually $\sigma_{\mathcal{A}_{p}}\left(p a^{*} a p\right) \subseteq(0, \infty)$. 
Now [Bi09, Corollary 4.7] shows that there exists a unique element $b=b^{*} \in \mathcal{A}_{p}$ such that $b^{2}=p a^{*} a p$ and $\sigma_{\mathcal{A}_{p}}(b) \subseteq[0, \infty)+\mathrm{i} \mathbb{R}$. Since $b^{2}=p a^{*} a p$, it follows (for instance by [Bi09, Remark 4.3]) that $\left\{z^{2} \mid z \in \sigma_{\mathcal{A}_{p}}(b)\right\}=\sigma_{\mathcal{A}_{p}}\left(p a^{*} a p\right) \subseteq(0, \infty)$. Now the property $\sigma_{\mathcal{A}_{p}}(b) \subseteq[0, \infty)+\mathrm{i} \mathbb{R}$ implies that $\sigma_{\mathcal{A}_{p}}(b) \subseteq(0, \infty)$. Thus, $b=b^{*} \in$ $\left(\mathcal{A}_{p}\right)^{\times}$and $p a^{*} a p=b^{2}$, as claimed.

To obtain the following statement we extend the method of proof of Proposition 3.1 in [Pi88].

Proposition 3.6. Let $\mathcal{A}$ be a Mackey-complete, hermitian algebra with continuous inversion and assume that $\delta: 0=p_{0}<p_{1}<\cdots<p_{n}=\mathbf{1}$ is a finite, totally ordered family of self-adjoint elements in $\mathcal{P}(\mathcal{A})$. Then for every $s \in \mathcal{A}^{\times}$there exist uniquely determined elements $d, b \in \mathcal{A}^{\times}$such that:

- $s^{*} s=b^{*} d b$;

- $\Phi_{\delta}(d)=d=d^{*}$ and $\sigma(d) \subseteq(0, \infty)$;

- $\Phi_{\delta}(b)=1$ and $b \in \Delta(\delta)^{\times}$.

PROOF. The case $n=1$ is clear: just take $b=\mathbf{1}$ and $d=s^{*} s$. Now assume that the conclusion holds for all families of at most $n$ self-adjoint idempotents in any Mackeycomplete, hermitian algebra with continuous inversion. Let $\delta$ be as in the statement and denote by $\delta_{n-1}$ the family $0=p_{0}<p_{1}<\cdots<p_{n-1}$ in $\mathcal{P}_{\mathcal{A}_{p_{n-1}}}$. Then $\mathcal{A}_{p_{n-1}}$ is a Mackey-complete, hermitian algebra with continuous inversion by Proposition 3.2, hence we can use Corollary 3.5 to obtain $y \in\left(\mathcal{A}_{p_{n-1}}\right)^{\times}$such that $p_{n-1} s^{*} s p_{n-1}=y^{*} y$. Then the induction hypothesis implies that there exist uniquely determined elements $b_{n-1}, d_{n-1} \in\left(\mathcal{A}_{p_{n-1}}\right)^{\times}$such that:

- $\quad p_{n-1} s^{*} s p_{n-1}=b_{n-1}^{*} d_{n-1} b_{n-1}$;

- $\quad \Phi_{\delta_{n-1}}\left(d_{n-1}\right)=d_{n-1}=d_{n-1}^{*}$ and $\sigma_{\mathcal{A}_{p_{n-1}}}\left(d_{n-1}\right) \subseteq(0, \infty)$;

- $\Phi_{\delta_{n-1}}\left(b_{n-1}\right)=p_{n-1}$ and $b_{n-1}, b_{n-1}^{-1} \in \Delta\left(\delta_{n-1}\right)$.

From now on we denote the elements in $\mathcal{A}$ as $2 \times 2$ matrices according to the decomposition $\mathbf{1}=p_{n-1}+\left(\mathbf{1}-p_{n-1}\right)$. For instance

$$
s^{*} s=\left(\begin{array}{cc}
p_{n-1} s^{*} s p_{n-1} & p_{n-1} s^{*} s\left(\mathbf{1}-p_{n-1}\right) \\
\left(\mathbf{1}-p_{n-1}\right) s^{*} s p_{n-1} & \left(\mathbf{1}-p_{n-1}\right) s^{*} s\left(\mathbf{1}-p_{n-1}\right)
\end{array}\right) .
$$

What we have to do is to find the still unknown entries in the matrices

$$
d=\left(\begin{array}{cc}
d_{n-1} & 0 \\
0 & d_{n}
\end{array}\right) \quad \text { and } \quad b=\left(\begin{array}{cc}
b_{n-1} & t_{n} \\
0 & \mathbf{1}-p_{n}
\end{array}\right)
$$

such that $s^{*} s=b^{*} d b$. By multiplying the corresponding matrices we see that the latter matrix equation is equivalent to the relations:

- $\quad p_{n-1} s^{*} s p_{n-1}=b_{n-1}^{*} d_{n-1} b_{n-1}$;

- $\quad p_{n-1} s^{*} s\left(\mathbf{1}-p_{n-1}\right)=b_{n-1}^{*} d_{n-1} t_{n} ;$ and

- $\quad\left(\mathbf{1}-p_{n-1}\right) s^{*} s\left(\mathbf{1}-p_{n-1}\right)=t_{n}^{*} d_{n-1} t_{n}+\left(\mathbf{1}-p_{n-1}\right) d_{n}\left(\mathbf{1}-p_{n-1}\right)$. 
We already know that the first of these equations is satisfied. Since $b_{n-1}$ and $d_{n-1}$ are invertible in $\mathcal{A}_{p_{n-1}}$ we can solve the second equation for $t_{n}$ to obtain

$$
t_{n}=d_{n-1}^{-1}\left(b_{n-1}^{*}\right)^{-1} p_{n-1} s^{*} s\left(\mathbf{1}-p_{n-1}\right) .
$$

Then the third of the above equations can be solved for $d_{n}$ and by using the above formula for $t_{n}$ we obtain

$$
\begin{aligned}
d_{n}= & \left(\mathbf{1}-p_{n-1}\right) s^{*} s\left(\mathbf{1}-p_{n-1}\right)-t_{n}^{*} d_{n-1} t_{n} \\
= & \left(\mathbf{1}-p_{n-1}\right) s^{*} s\left(\mathbf{1}-p_{n-1}\right)-\left(\left(\mathbf{1}-p_{n-1}\right) s^{*} s p_{n-1} b_{n-1}^{-1} d_{n-1}^{-1}\right) d_{n-1} \\
& \times \underbrace{\left(d_{n-1}^{-1}\left(b_{n-1}^{*}\right)^{-1} p_{n-1} s^{*} s\left(\mathbf{1}-p_{n-1}\right)\right)}_{=t_{n}} \\
= & \left(\mathbf{1}-p_{n-1}\right)\left(s^{*} s-s^{*} s p_{n-1} b_{n-1}^{-1} d_{n-1}^{-1}\left(b_{n-1}^{*}\right)^{-1} p_{n-1} s^{*} s\right)\left(\mathbf{1}-p_{n-1}\right) .
\end{aligned}
$$

Since $p_{n-1} s^{*} s p_{n-1}=b_{n-1}^{*} d_{n-1} b_{n-1}$, we obtain the following formula for $d_{n}$ in terms of $s^{*} s$ and $p_{n-1}$ only:

$$
d_{n}=\left(\mathbf{1}-p_{n-1}\right)\left(s^{*} s-s^{*} s p_{n-1}\left(p_{n-1} s^{*} s p_{n-1}\right)^{-1} p_{n-1} s^{*} s\right)\left(\mathbf{1}-p_{n-1}\right) .
$$

The induction step is complete. Note that the property $\sigma(d) \subseteq(0, \infty)$ follows by the theorem of Shirali-Ford type for Mackey-complete, hermitian algebras with continuous inversion [Bi09, Corollary 7.7] since $b$ is clearly invertible and $d=$ $\left(b^{*}\right)^{-1} s^{*} s b^{-1}$. The uniqueness assertion is straightforward. See [Pi88, Proof of Proposition 3.1] for further details which carry over in a direct manner to the present setting.

Corollary 3.7. Let $\mathcal{A}$ be a Mackey-complete, hermitian algebra with continuous inversion and assume that $\delta: 0=p_{0}<p_{1}<\cdots<p_{n}=\mathbf{1}$ is a finite, totally ordered family of self-adjoint elements in $\mathcal{P}(\mathcal{A})$. Then, for every $s \in \mathcal{A}^{\times}$, there exist uniquely determined elements $u \in \mathrm{U}(\mathcal{A}), a \in D(\delta)_{+}^{\times}$and $b \in \Delta(\delta)^{\times}$such that $\Phi_{\delta}(b)=\mathbf{1}$ and $s=u a b$.

PROOF. It follows by Proposition 3.6 that $s^{*} s=b^{*} d b$, where $d$ and $b$ are uniquely determined by the conditions $d \in D(\delta)_{+}^{\times}, b \in \Delta(\delta)^{\times}$, and $\Phi_{\delta}(b)=1$. Define $a=$ $d^{1 / 2} \in \operatorname{Ran}\left(\Phi_{\delta}\right)_{+}^{\times}$by Corollary 4.7 along with [Bi09, Proposition 7.10], since $D(\delta)$ $\left(=\operatorname{Ran}\left(\Phi_{\delta}\right)\right)$ is a closed unital $*$-subalgebra of $\mathcal{A}$. Then $s^{*} s=(a b)^{*}(a b)$, whence $\left(s(a b)^{-1}\right)^{*}\left(s(a b)^{-1}\right)=\mathbf{1}$. Thus, $u:=s(a b)^{-1} \in \mathrm{U}(\mathcal{A})$ and $s=u a b$.

For the uniqueness assertion assume that $s=u^{\prime} a^{\prime} b^{\prime}$ is another decomposition with similar properties. Then $b^{*} a^{\prime 2} b^{\prime}=s^{*} s=b^{*} a^{2} b$, whence $b=b^{\prime}$ and $a=a^{\prime}$ according to the uniqueness property from the above Proposition 3.6 along with the uniqueness of nonnegative square roots.

REMARK 3.8. It follows by the explicit construction performed in the proofs of Corollary 3.7 and Proposition 3.6 that there actually exist real analytic mappings 
$u(\cdot), a(\cdot), b(\cdot): \mathcal{A}^{\times} \rightarrow \mathcal{A}$ such that $u(\cdot)$ takes values in $\mathrm{U}(\mathcal{A}), a(\cdot)$ takes values in $D(\delta)_{+}^{\times}$, and $b(\cdot)$ takes values in $N(\delta)$, and

$$
\left(\forall s \in \mathcal{A}^{\times}\right) \quad s=u(s) a(s) b(s) .
$$

Therefore, by using an appropriate real analytic structure on $\mathrm{U}(\mathcal{A})$ constructed by means of the Cayley transform (see [BrN05, Section 8]), it follows that the multiplication mapping

$$
\mathrm{U}(\mathcal{A}) \times D(\delta)_{+}^{\times} \times N(\delta) \rightarrow \mathcal{A}^{\times}, \quad(u, a, b) \mapsto u a b
$$

is a real analytic diffeomorphism.

\section{Flag manifolds}

We are now ready to obtain the main results of the present paper: the construction of appropriate smooth structures on the flag manifolds associated with algebras with continuous inversion (Theorem 4.3) and the characterization of the hermitian algebras in terms of transitivity of unitary group actions on flag manifolds (Theorem 4.5 and Corollary 4.7).

Definition 4.1. Let $\mathcal{A}$ be a complex associative unital algebra. For $p, q \in \mathcal{P}(\mathcal{A})$ we use the notation $p \sim q$ if and only if $p q=q$ and $q p=p$, which is easily seen to be equivalent to $p \mathcal{A}=q \mathcal{A}$. This is an equivalence relation and we denote the equivalence class of $p \in \mathcal{P}(\mathcal{A})$ by $[p]$.

Clearly, each $p \mathcal{A}$ is a complemented right ideal of $\mathcal{A}$ and, conversely, if $\mathcal{R} \subseteq \mathcal{A}$ is a complemented right ideal, then any $\mathcal{A}$-right module projection $\mathcal{A} \rightarrow \mathcal{R}$ is given by a left multiplication with an idempotent $p$, satisfying $\mathcal{R}=p \mathcal{A}$. Therefore, the Graßmannian $\operatorname{Gr}(\mathcal{A})=\mathcal{P}(\mathcal{A}) / \sim$ of $\mathcal{A}$ can be identified with the set of complemented right ideals of $\mathcal{A}$ (see [BrN04, Section 8.6]).

If $p_{1}, p_{2} \in \mathcal{P}(\mathcal{A})$, recall that we write $p_{1} \leq p_{2}$ whenever $p_{2} p_{1}=p_{1}$, that is, $p_{1} \mathcal{A} \subseteq p_{2} \mathcal{A}$. In addition, let us recall from [B1G09, Lemma 2.2] that the natural action $\alpha: \mathcal{A}^{\times} \times \mathcal{P}(\mathcal{A}) \rightarrow \mathcal{P}(\mathcal{A}),(g, p) \mapsto \alpha_{g}(p):=g p g^{-1}$, induces an action $\beta(g, \mathcal{R}):=$ $g \mathcal{R}$ of $\mathcal{A}^{\times}$on the set $\operatorname{Gr}(\mathcal{A})$ of complemented right ideals. Clearly, this action preserves the inclusion order on $\operatorname{Gr}(\mathcal{A})$ and hence on $\mathcal{P}(\mathcal{A})$.

For every $n \geq 1$, we define the set of $n$-flags in a similar manner as above. For this purpose, first denote

$$
\mathcal{P}_{n}(\mathcal{A})=\left\{\left(p_{1}, \ldots, p_{n}\right) \in \mathcal{P}(\mathcal{A}) \times \cdots \times \mathcal{P}(\mathcal{A}) \mid p_{1} \leq \cdots \leq p_{n}\right\}
$$

and define an equivalence relation $\sim$ on $\mathcal{P}_{n}(\mathcal{A})$ by $\left(p_{1}, \ldots, p_{n}\right) \sim\left(q_{1}, \ldots, q_{n}\right)$ if and only if $\left[p_{j}\right]=\left[q_{j}\right]$ for $j=1, \ldots, n$; the equivalence class of $\left(p_{1}, \ldots, p_{n}\right)$ will be denoted by $\left[\left(p_{1}, \ldots, p_{n}\right)\right]$. Now the set of $n$-flags is $\mathrm{Fl}_{\mathcal{A}}(n)=\mathcal{P}_{n}(\mathcal{A}) / \sim$. There exists a natural injective map

$$
\mathrm{Fl}_{\mathcal{A}}(n) \hookrightarrow \operatorname{Gr}(\mathcal{A})^{n}, \quad\left[\left(p_{1}, \ldots, p_{n}\right)\right] \mapsto\left(p_{1} \mathcal{A}, \ldots, p_{n} \mathcal{A}\right)
$$


whose image consists of all $n$-tuples $\left(R_{1}, \ldots, R_{n}\right)$ of complemented right ideals satisfying $R_{1} \leq \cdots \leq R_{n}$. This subset is invariant under the natural action by left multiplication $\beta^{(n)}: \mathcal{A}^{\times} \times \mathrm{Fl}_{\mathcal{A}}(n) \rightarrow \mathrm{Fl}_{\mathcal{A}}(n)$. For every $X \in \mathrm{Fl}_{\mathcal{A}}(n)$, the corresponding flag manifold $\mathrm{Fl}_{\mathcal{A}}(X)$ is defined as the $\mathcal{A}^{\times}$-orbit of $X$.

Smooth structure on the flag manifolds If $\mathcal{A}$ is a CIA, the manifold structure on the corresponding Graßmannian was pointed out in [BrN05, Theorem 5.3]; see also [DG01, Remark 7.1] for the Banach case. In the case of the flag manifolds associated with a CIA, to construct the smooth structure one can proceed as follows.

REMARK 4.2. Let $\mathcal{A}$ be a CIA. If $\delta: 0=p_{0}<p_{1}<\cdots<p_{n}=\mathbf{1}$ is a finite, totally ordered family of elements in $\mathcal{P}(\mathcal{A})$, then one proceeds by induction on $n$ to show that $g \in \mathcal{A}^{\times}$has the property that $p_{j} g p_{j} \in\left(p_{j} \mathcal{A} p_{j}\right)^{\times}$for $j=1, \ldots, n$ if and only if there exists a (uniquely determined) Gau $\beta$ decomposition

$$
g=x d y, \quad \text { where } d \in D(\delta)^{\times}, x \in N(\mathbf{1}-\delta) \text { and } y \in N(\delta),
$$

where we denote by $\mathbf{1}-\delta$ the sequence $0=\mathbf{1}-p_{n}<\mathbf{1}-p_{n-1}<\cdots<\mathbf{1}-p_{0}=\mathbf{1}$. For instance, let us denote the elements of $\mathcal{A}$ as $2 \times 2$ matrices according to the decomposition $\mathbf{1}=p_{n-1}+\left(\mathbf{1}-p_{n-1}\right)$ as in the proof of Proposition 3.6. For every $g \in \mathcal{A}^{\times}$such that $p_{n-1} g p_{n-1} \in\left(p_{n-1} \mathcal{A} p_{n-1}\right)^{\times}$we have

$$
\begin{aligned}
g= & \left(\begin{array}{cc}
p_{n-1} g p_{n-1} & p_{n-1} g\left(\mathbf{1}-p_{n-1}\right) \\
\left(\mathbf{1}-p_{n-1}\right) g p_{n-1} & \left(\mathbf{1}-p_{n-1}\right) g\left(\mathbf{1}-p_{n-1}\right)
\end{array}\right) \\
= & \left(\begin{array}{cc}
p_{n-1} & 0 \\
x_{n-1} & \mathbf{1}-p_{n-1}
\end{array}\right)\left(\begin{array}{cc}
p_{n-1} g p_{n-1} & 0 \\
0 & \left(\mathbf{1}-p_{n-1}\right) g\left(\mathbf{1}-p_{n-1}\right)
\end{array}\right) \\
& \times\left(\begin{array}{cc}
p_{n-1} & y_{1} \\
0 & \mathbf{1}-p_{n-1}
\end{array}\right)
\end{aligned}
$$

where, if we denote by $\left(p_{n-1} g p_{n-1}\right)^{-1}$ the inverse of $p_{n-1} g p_{n-1}$ in the algebra $p_{n-1} \mathcal{A} p_{n-1}$, then

$$
x_{n-1}=\left(\mathbf{1}-p_{n-1}\right) g p_{n-1}\left(p_{n-1} g p_{n-1}\right)^{-1}
$$

and

$$
y_{1}=\left(p_{n-1} g p_{n-1}\right)^{-1} p_{n-1} g\left(\mathbf{1}-p_{n-1}\right) .
$$

Similar computations performed in the algebras $p_{n-1} \mathcal{A} p_{n-1}, \ldots, p_{2} \mathcal{A} p_{2}$ eventually lead to the decomposition (4.2) under the corresponding assumption on the element $g \in \mathcal{A}^{\times}$. Denote

$$
\Omega_{\delta}=\left\{g \in \mathcal{A}^{\times} \mid p_{j} g p_{j} \in\left(p_{j} \mathcal{A} p_{j}\right)^{\times} \text {for } j=1, \ldots, n\right\}
$$

and define

$$
\sigma: \Omega_{\delta} \rightarrow \mathcal{A}, \quad g \mapsto x
$$

by means of the decomposition (4.2). It is clear from the construction that $\sigma$ is a real analytic mapping on the neighborhood $\Omega_{\delta}$ of $1 \in \mathcal{A}^{\times}$. 
THEOREM 4.3. If $\mathcal{A}$ is a CIA and $[\delta]:=\left[\left(p_{1}, \ldots, p_{n}\right)\right] \in \mathrm{Fl}_{\mathcal{A}}(n)$, then the corresponding manifold $\mathrm{Fl}_{\mathcal{A}}([\delta])$ has a structure of smooth manifold modeled on a locally convex space such that the transitive action

$$
\left.\beta^{(n)}\right|_{\mathcal{A}^{\times} \times \mathrm{Fl}_{\mathcal{A}}([\delta])}: \mathcal{A}^{\times} \times \mathrm{Fl}_{\mathcal{A}}([\delta]) \rightarrow \mathrm{Fl}_{\mathcal{A}}([\delta])
$$

is smooth, and the corresponding orbit mapping $\mathcal{A}^{\times} \rightarrow \mathrm{Fl}_{\mathcal{A}}([\delta]), g \mapsto \beta^{(n)}(g,[\delta])$ is smooth and open. Moreover, the injective map $\operatorname{Fl}_{\mathcal{A}}(n) \hookrightarrow \operatorname{Gr}(\mathcal{A})^{n}$ (see (4.1)) is continuous.

Proof. By definition, the flag manifold $\mathrm{Fl}_{\mathcal{A}}([\delta])$ is transitively acted on by the group $\mathcal{A}^{\times}$, and the stabilizer of $[\delta]=\left(p_{1} \mathcal{A}, \ldots, p_{n} \mathcal{A}\right)$ is

$$
B:=\left\{g \in \mathcal{A}^{\times} \mid g, g^{-1} \in \Delta(\delta)\right\}=\Delta(\delta)^{\times},
$$

which is a closed subgroup of $\mathcal{A}^{\times}$. We thus obtain a bijection

$$
\mathrm{Fl}_{\mathcal{A}}([\delta]) \simeq \mathcal{A}^{\times} / \Delta(\delta)^{\times} .
$$

Moreover, it is clear that $\Delta(\delta)^{\times}=D(\delta)^{\times} N(\delta)$ and it easily follows by the method that the Gauß decomposition was constructed in Remark 4.2 above that the multiplication mapping $N(\mathbf{1}-\delta) \times \Delta(\delta)^{\times} \rightarrow \Omega_{\delta}$ is a homeomorphism. Since $\mathcal{A}^{\times}$is a locally convex Lie group (see [G102]), it then follows that a natural smooth structure on the flag manifold $\mathrm{Fl}_{\mathcal{A}}([\delta])$ can be constructed by using Lemma A.1 in Appendix A.

Note that Lemma A.1 also implies that this smooth structure depends only on the point $[\delta] \in \mathrm{Fl}_{\mathcal{A}}(n)$ and not on the choice of $\left(p_{1}, \ldots, p_{n}\right) \in[\delta]$, since for any other $\delta^{\prime}=\left(p_{1}^{\prime}, \ldots, p_{n}^{\prime}\right) \in[\delta]$, the subgroup $N\left(\mathbf{1}-\delta^{\prime}\right)$ leads to the same smooth structure.

Finally, we recall that the set $\mathcal{P}(\mathcal{A})$ is endowed with the topology induced from $\mathcal{A}$, and then the $\operatorname{Graßmannian} \operatorname{Gr}(\mathcal{A})=\mathcal{P}(\mathcal{A}) / \sim$ is endowed with the corresponding quotient topology. By using the special case $n=1$ of the construction above, one can see that the quotient mapping $\mathcal{P}(\mathcal{A}) \rightarrow \operatorname{Gr}(\mathcal{A})$ is open. Now it is easy to see that the natural mapping (4.1) from $\mathrm{Fl}_{\mathcal{A}}([\delta]) \rightarrow \operatorname{Gr}(\mathcal{A})^{n}$ is continuous with respect to the above-described manifold structure on $\mathrm{Fl}_{\mathcal{A}}([\delta])$ and the product topology on $\operatorname{Gr}(\mathcal{A})^{n}$.

Unitary groups acting on the flag manifolds The second assertion in the following lemma was recorded with the same idea of proof in [MS97, Lemma 1.3] for $C^{*}$ algebras. We include here the full details of the proof in order to show that the corresponding statement is actually purely algebraic.

Lemma 4.4. If $\mathcal{A}$ is a complex associative unital algebra, then the following assertions hold.

(1) If $p, q \in \mathcal{P}(\mathcal{A})$ and $p \sim q$, then $s:=p q+(\mathbf{1}-p)(\mathbf{1}-q)$ satisfies $s \in \mathcal{A}^{\times}$and $s q s^{-1}=p$. 
(2) If $\mathcal{A}$ is additionally endowed with an involution that makes it into a hermitian algebra, then for every $e \in \mathcal{P}(\mathcal{A})$, there exists a unique $p \in \mathcal{P}(\mathcal{A})$ such that $p=p^{*}$ and $p \sim e$, namely $p=e\left(\mathbf{1}-\left(e^{*}-e\right)\right)^{-1}$.

Proof. (1) To see that $s$ is invertible, recall that $p \sim q$ means $p q=q$ and $q p=p$, which easily yields $(q-p)^{2}=0$ and $s=\mathbf{1}+(q-p)$, and then $s \in \mathcal{A}^{\times}$with $s^{-1}=$ $\mathbf{1}-(q-p)$. The relation $s q s^{-1}=p$ follows immediately from $s q=p q=p s$.

(2) First note that the element $p \in \mathcal{A}$ in the statement is well defined since $\left(e^{*}-e\right)^{*}=-\left(e^{*}-e\right)$, hence the hypothesis that $\mathcal{A}$ is a hermitian algebra ensures that the number $1 \in \mathbb{C}$ does not belong to the spectrum of $e^{*}-e \in \mathcal{A}$. Next, in order to prove the existence assertion, we have to check that the element $p \in \mathcal{A}$ has the required properties

$$
\text { ep }=p, \quad p e=e, \quad \text { and } \quad p=p^{*}=p^{2} .
$$

The first of these equations follows at once since $e^{2}=e$. The latter equality also implies $\left(e^{*}\right)^{2}=e^{*}$, whence $e^{*}\left(\mathbf{1}-\left(e^{*}-e\right)\right)=e^{*} e=\left(\mathbf{1}+\left(e^{*}-e\right)\right) e$. Then we get $\left(\mathbf{1}+\left(e^{*}-e\right)\right)^{-1} e^{*}=e\left(\mathbf{1}-\left(e^{*}-e\right)\right)^{-1}$, that is, $p^{*}=p$. Moreover, since $p\left(1-\left(e^{*}-e\right)\right)=e$, we obtain $p e-e=p e^{*}-p$, hence $p e-e=\left(e p^{*}-p^{*}\right)^{*}=0$, since we have just seen that $p^{*}=p$ and $e p=p$. Thus, $p e=e$, and then $p e\left(\mathbf{1}-\left(e^{*}-e\right)\right)^{-1}=e\left(\mathbf{1}-\left(e^{*}-e\right)\right)^{-1}$, that is, $p^{2}=p$. Consequently, $p=p^{*} \in$ $\mathcal{P}(\mathcal{A})$ and $p \sim e$.

For the uniqueness assertion, assume that $q=q^{*} \in \mathcal{P}(\mathcal{A})$ and $q \sim e$. In particular, $e q=q$, whence $q^{*} e^{*}=q^{*}$, so $q e^{*}=q$. Since also $q e=e$, by subtracting these equalities from each other we obtain $q\left(e^{*}-e\right)=q-e$. Thence $q\left(\mathbf{1}-\left(e^{*}-e\right)\right)=e$, and then $q=e\left(\mathbf{1}-\left(e^{*}-e\right)\right)^{-1}=p$. This completes the proof.

THEOREM 4.5. If $\mathcal{A}$ is a Mackey-complete, hermitian CIA, then the corresponding flag manifolds are transitively acted on by the unitary group of $\mathcal{A}$.

PROOF. Let $\left(p_{1}, \ldots, p_{n}\right) \in \mathcal{P}_{n}(\mathcal{A})$. We prove that the unitary group of $\mathcal{A}$ acts transitively on the flag manifold $\mathrm{Fl}_{\mathcal{A}}\left(\left[\left(p_{1}, \ldots, p_{n}\right)\right]\right)$. By Lemma 4.4(2), we may assume that $p_{j}=p_{j}^{*}$ for $j=1, \ldots, n$. Then $\delta: 0=p_{0}<p_{1}<\cdots<p_{n}<p_{n+1}=\mathbf{1}$ will be a finite, totally ordered family of self-adjoint elements in $\mathcal{P}_{\mathcal{A}}$. Now let $g \in \mathcal{A}^{\times}$ be arbitrary. We have to prove that there exists a $u \in \mathrm{U}(\mathcal{A})$ such that for $j=1, \ldots, n$ we have $g p_{j} \mathcal{A}=u p_{j} \mathcal{A}$. For this purpose we may use the element $u \in \mathrm{U}(\mathcal{A})$ provided by Corollary 3.7, since the factors in the corresponding decomposition $g=u a b$ satisfy $a b p_{j} \mathcal{A}=p_{j} \mathcal{A}$, hence $g p_{j} \mathcal{A}=u p_{j} \mathcal{A}$, and this completes the proof.

REMARK 4.6. The above Theorem 4.5 is a wide extension of [B1R07, Proposition 2.7], whose method of proof is specific for finite $W^{*}$-algebras.

Corollary 4.7. Let $\mathcal{A}$ be a Mackey-complete CIA. Then $\mathcal{A}$ is hermitian if and only if the matrix algebra $M_{2}(\mathcal{A})$ has the property that each of the corresponding flag manifolds is acted transitively on by the unitary group $\mathrm{U}_{2}(\mathcal{A})$. 
PROOF. If $\mathcal{A}$ is hermitian, then the Mackey-complete CIA $M_{2}(\mathcal{A})$ is hermitian by Proposition 3.3, hence Theorem 4.5 shows that the unitary group $\mathrm{U}_{2}(\mathcal{A})$ of $M_{2}(\mathcal{A})$ acts transitively on every flag manifold of $M_{2}(\mathcal{A})$.

Conversely, if that transitivity condition is satisfied, then $\mathcal{A}$ has to be a hermitian algebra. Indeed, if $\mathcal{A}$ is not hermitian, then Remark 2.3 shows that there exists a hermitian element $a=a^{*} \in \mathcal{A}$ with $\mathrm{i} \in \sigma(a)$, so that $a^{2}+\mathbf{1}$ is not invertible. Now let $\delta: 0=p_{0}<p_{1}<p_{2}=\mathbf{1}$ in $M_{2}(\mathcal{A})$, where

$$
p_{1}=\left(\begin{array}{ll}
\mathbf{1} & 0 \\
0 & 0
\end{array}\right)
$$

and note that the matrix

$$
g=\left(\begin{array}{ll}
\mathbf{1} & 0 \\
a & \mathbf{1}
\end{array}\right) \in \mathrm{GL}_{2}(\mathcal{A})
$$

is not contained in the subset $\mathrm{U}_{2}(\mathcal{A}) \Delta(\delta)^{\times}$because the entry $\left(g^{*} g\right)_{11}=\mathbf{1}+a^{2}$ is not invertible. It thus follows that the element $\beta^{(2)}(g,[\delta]) \in \mathrm{Fl}_{\mathcal{A}}([\delta])$ is different from $\beta^{(2)}(u,[\delta])$ for every $u \in \mathrm{U}_{2}(\mathcal{A})$, and this contradicts the assumption that $\mathrm{U}_{2}(\mathcal{A})$ acts transitively on every flag manifold of $M_{2}(\mathcal{A})$.

REMARK 4.8. We also note that the matrix

$$
g=\left(\begin{array}{cc}
a+\mathrm{i} & a \\
a & a-\mathrm{i}
\end{array}\right) \in \mathrm{GL}_{2}(\mathcal{A})
$$

satisfies $g J g^{*} J=\mathbf{1}$ for

$$
J:=\left(\begin{array}{rr}
1 & 0 \\
0 & -1
\end{array}\right),
$$

so that

$$
g \in \mathrm{U}_{1,1}(\mathcal{A}):=\left\{g \in \mathrm{GL}_{2}(A) \mid g^{-1}=J g^{*} J\right\} .
$$

Since $(a+\mathrm{i})(a-\mathrm{i})=a^{2}+\mathbf{1}$ implies that the entry $g_{11}=a+i$ is not invertible, it follows that $g$ is not contained in the open subset

$$
N(\mathbf{1}-\delta) \Delta(\delta)^{\times} .
$$

If, conversely, $(\mathcal{A}, *)$ is hermitian, then

$$
g=\left(\begin{array}{ll}
a & b \\
c & d
\end{array}\right) \in \mathrm{U}_{1,1}(\mathcal{A})
$$

implies that $a a^{*}=\mathbf{1}+b b^{*}$ is invertible, so that $a \in \mathcal{A}^{\times}$, which implies that

$$
\mathrm{U}_{1,1}(\mathcal{A}) \subseteq N(\mathbf{1}-\delta) \Delta(\delta)^{\times}
$$

(see [Bi09]). 


\section{Acknowledgements}

The most substantial part of this paper was written during a visit of the first-named author at the Department of Mathematics of TU Darmstadt. Financial support and the excellent working conditions provided there are gratefully acknowledged. The same author also acknowledges partial financial support from the grant PNII-Programme 'Idei' (code 1194).

\section{Appendix A. A lemma on manifold structures on homogeneous spaces}

The following statement is a more precise version, in the present setting, of [DG00, Corollary 5.3]. It equally applies to $\mathcal{C}^{\infty}$ and to real analytic manifolds modeled on locally convex spaces.

LEMMA A.1. Let $G$ be a locally convex Lie group and assume that $B$ is a closed subgroup of $G$ for which there exists a subset $N \subseteq G$ such that the following conditions are satisfied:

(1) $\mathbf{1} \in N$;

(2) $N$ is homeomorphic to an open set in some locally convex space, and that homeomorphism makes $N$ into a manifold such that the inclusion $N \hookrightarrow G$ is a smooth mapping;

(3) there exists an open neighborhood $\Omega$ of $\mathbf{1} \in G$ such that the multiplication mapping

$$
N \times B \rightarrow \Omega, \quad(n, b) \rightarrow n b
$$

is a homeomorphism and the corresponding projection mapping $\sigma: \Omega \rightarrow N$, $n b \mapsto n$ is smooth.

Then the homogeneous space $G / B$ endowed with the quotient topology has a structure of manifold (with the same model space as $N$ ) such that the natural projection $\pi: G \rightarrow G / B$ is smooth and open, and the natural action

$$
G \times G / B \rightarrow G / B, \quad(g, n B) \mapsto L_{g}(n B):=g n B
$$

is smooth.

Moreover, if $N^{\prime} \subseteq G$ is another subset satisfying (1)-(3), then it defines the same smooth structure on $G / B$.

PROOF. Let us define

$$
\psi_{\mathbf{1}}:=\left.\pi\right|_{N}: N \rightarrow G / B \quad \text { and } \quad V_{\mathbf{1}}:=\psi_{\mathbf{1}}(N) \subseteq G / B .
$$

Note that $\psi_{\mathbf{1}}$ is injective as if $\psi_{\mathbf{1}}\left(n_{1}\right)=\psi_{\mathbf{1}}\left(n_{2}\right)$, then $n_{1} \in n_{2} B$, hence $n_{1}=n_{2}$ because of the hypothesis that the multiplication mapping $N \times B \rightarrow \Omega$ bijective. Next, for $g \in G$, define

$$
\psi_{g}:=\left.L_{g} \circ \pi\right|_{N}: N \rightarrow G / B \quad \text { and } \quad V_{g}:=\psi_{g}(N) \subseteq G / B .
$$


It is clear that $G / N=\bigcup_{g \in G} V_{g}$ so, in order for the family of bijections $\left\{\psi_{g}: N \rightarrow V_{g}\right\}_{g \in G}$ to define a structure of smooth manifold on $G / B$, we still have to prove that for every $g \in G$ the set $\psi_{g}^{-1}\left(V_{g} \cap V_{\mathbf{1}}\right)$ is open in $N$ and the coordinate change $\left.\psi_{\mathbf{1}}^{-1} \circ \psi_{g}\right|_{\psi_{g}^{-1}\left(V_{g} \cap V_{\mathbf{1}}\right)}: \psi_{g}^{-1}\left(V_{g} \cap V_{\mathbf{1}}\right) \rightarrow \psi_{\mathbf{1}}^{-1}\left(V_{g} \cap V_{\mathbf{1}}\right)$ is smooth. In fact, for $x \in \psi_{g}^{-1}\left(V_{g} \cap V_{\mathbf{1}}\right) \subseteq N$ and $x^{\prime} \in \psi_{\mathbf{1}}^{-1}\left(V_{g} \cap V_{\mathbf{1}}\right) \subseteq N$ we have

$$
\begin{aligned}
\left(\psi_{1}^{-1} \circ \psi_{g}\right)(x)=x^{\prime} & \Longleftrightarrow \psi_{g}(x)=\psi_{\mathbf{1}}\left(x^{\prime}\right) \Longleftrightarrow g x B=x^{\prime} B \\
& \Longleftrightarrow g x \in x^{\prime} B \Longleftrightarrow g x \in \Omega
\end{aligned}
$$

and

$$
x^{\prime}=\sigma(g x)
$$

and therefore $\psi_{g}^{-1}\left(V_{g} \cap V_{\mathbf{1}}\right)=\left(g^{-1} \Omega\right) \cap N$ is open in $N$ and

$$
\psi_{\mathbf{1}}^{-1} \circ \psi_{g}:\left(g^{-1} \Omega\right) \cap N \rightarrow N, \quad x \mapsto \sigma(g x)
$$

is smooth because of the hypothesis on $N$.

Thus, we obtain a manifold structure on $G / B$ such that the translation mapping $L_{g}: G / B \rightarrow G / B$ is smooth for every $g \in G$. Since $\psi_{\mathbf{1}}: V_{\mathbf{1}} \rightarrow N \hookrightarrow G$ is a continuous cross section of $\pi: G \rightarrow G / B$ over the open subset $V_{1}$ of the manifold $G / B$, it follows that the projection $\pi$ is an open mapping. Moreover, since the multiplication mapping $N \times B \rightarrow \Omega$ is a bijection, we obtain $\pi(\Omega)=\pi(N)=$ $V_{1} \subseteq G / B$, and then it easily follows that $\pi: G \rightarrow G / B$ is continuous. Since $\pi$ is also open, the topology underlying the manifold structure of $G / B$ coincides with the quotient topology. As $B$ is a closed subgroup of $G$, the corresponding topology of $G / B$ is Hausdorff.

It is clear from the above construction that (A1) is an action of $G$ by smooth transforms on $G / B$. Therefore, for proving that (A1) is actually a smooth mapping, it is enough to check that it is smooth on some neighborhood of the point $(\mathbf{1}, \mathbf{1} B) \in$ $G \times G / B$. To this end, let $U$ be an open neighborhood of $1 \in G$ and $V_{1}^{\prime}$ an open neighborhood of $\mathbf{1} B \in B / B$ such that $L_{g}(x) \in V_{\mathbf{1}}$ for all $g \in U$ and $x \in V_{\mathbf{1}}^{\prime}$. Then we have a commutative diagram

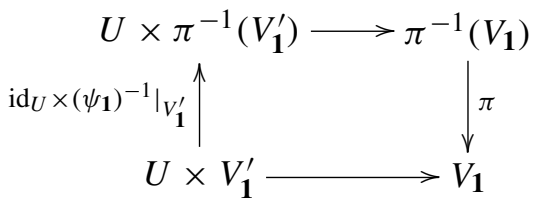

whose upper horizontal arrow is given by the multiplication in $G$, while the lower horizontal arrow is the appropriate restriction of the mapping (A1). Thus, the latter restriction of the group action (A1) factorizes as a composition of smooth mappings, and it is therefore smooth on the neighborhood $U \times V_{\mathbf{1}}^{\prime}$ of the point $(\mathbf{1}, \mathbf{1} B) \in G \times$ $G / B$. 
Finally, suppose that $N^{\prime} \subseteq G$ is another subset satisfying (1)-(3). If $N^{\prime}$ is an open subset of $N$, we clearly obtain the same manifold structure on $G / B$ because $G$ acts by diffeomorphisms, so that it is determined by the smooth structure in a neighborhood of $\pi(\mathbf{1})$. In the general case, the subset $\widetilde{N}^{\prime}:=N^{\prime} \cap \Omega$ is open in $N^{\prime}$, and (3) implies that $N^{\prime \prime} B$ is open in $G$, so that $\widetilde{N}:=N \cap \tilde{N}^{\prime} B$ is open in $N$. Passing from $N$ to $\widetilde{N}$ and from $N^{\prime}$ to $\tilde{N}^{\prime}$, we may therefore assume that $N B=N^{\prime} B$. Now (3) implies that the map $N^{\prime} \rightarrow N, n^{\prime} \mapsto \sigma\left(n^{\prime}\right)$ is a diffeomorphism whose inverse is given by $N \rightarrow N^{\prime}, n \mapsto \sigma^{\prime}(n)$. From that we conclude that the map $\psi_{1}^{\prime} \circ \psi_{1}: N B \rightarrow N^{\prime} B$ is a diffeomorphism, which in turn implies that $N$ and $N^{\prime}$ define the same smooth structure on $G / B$.

\section{References}

[B106] D. Beltiţă, Smooth Homogeneous Structures in Operator Theory, Monographs and Surveys in Pure and Applied Mathematics, 137 (Chapman and Hall/CRC Press, Boca Raton, FL, 2006).

[BIG09] D. Beltiţă and J. E. Galé, 'On complex infinite-dimensional Grassmann manifolds', Complex Anal. Oper. Theory to appear.

[BIN08] D. Beltiţă and K.-H. Neeb, 'Finite-dimensional Lie subalgebras of algebras with continuous inversion', Studia Math. 185 (2008), 249-262.

[BIR07] D. Beltiţă and T. S. Ratiu, 'Geometric representation theory for unitary groups of operator algebras', Adv. Math. 208 (2007), 299-317.

[BrN04] W. Bertram and K.-H. Neeb, 'Projective completions of Jordan pairs. I. The generalized projective geometry of a Lie algebra', J. Algebra 277 (2004), 474-519.

[BrN05] - 'Projective completions of Jordan pairs. II. Manifold structures and symmetric spaces', Geom. Dedicata 112 (2005), 73-113.

[Bi09] H. Biller, 'Continuous inverse algebras with involution', Forum Math. to appear.

[DG00] M. J. Dupré and J. F. Glazebrook, 'Infinite dimensional manifold structures on principal bundles', J. Lie Theory 10 (2000), 359-373.

[DG01] _ 'The Stiefel bundle of a Banach algebra', Integral Equations Operator Theory 41 (2001), 264-287.

[FGL06] G. Fendler, K. Gröchenig and M. Leinert, 'Symmetry of weighted $L^{1}$-algebras and the GRS-condition', Bull. London Math. Soc. 38 (2006), 625-635.

[G102] H. Glöckner, 'Algebras whose groups of units are Lie groups', Studia Math. 153 (2002), 147-177.

[GIN09] H. Glöckner and K.-H. Neeb, Infinite-dimensional Lie Groups, Springer, 2009, in preparation.

[Ko69] G. Köthe, Topological Vector Spaces I, Grundlehren der Mathematischen Wissenschaften, 159 (Springer, Berlin, 1969).

[Ku79] W. Kugler, 'On the symmetry of generalized $L^{1}$-algebras', Math. Z. 168 (1979), 241-262.

[Le76] H. Leptin, 'Symmetrie in Banachschen Algebren', Arch. Math. (Basel) 27 (1976), 394-400.

[MS97] M. Martin and N. Salinas, 'Flag manifolds and the Cowen-Douglas theory', J. Operator Theory 38 (1997), 329-365.

[MNS09] C. Müller, K.-H. Neeb and H. Seppänen, 'Borel-Weil theory for root graded Banach-Lie groups', Preprint, arXiv:0903.1188v1.

[Ne03] K.-H. Neeb, 'Locally convex root graded Lie algebras', Trav. Math. 14 (2003), 25-120.

[Ne06] _ 'Towards a Lie theory of locally convex groups', Jpn. J. Math. 1 (2006), 291-468.

[Ne08] _ ' 'Lie group extensions associated to projective modules of continuous inverse algebras', Arch. Math. (Brno) 44 (2008), 339-363. 
[Pi88] D. R. Pitts, 'Factorization problems for nests: factorization methods and characterizations of the universal factorization property', J. Funct. Anal. 79 (1988), 57-90.

[Po77] D. Poguntke, 'Nilpotente Liesche Gruppen haben symmetrische Gruppenalgebren', Math. Ann. 227 (1977), 51-59.

[PS90] A. Pressley and G. Segal, Loop Groups, Oxford Mathematical Monographs (Oxford University Press, Oxford, 1990).

[Sw77] R. G. Swan, 'Topological examples of projective modules', Trans. Amer. Math. Soc. 230 (1977), 201-234.

[Up85] H. Upmeier, Symmetric Banach Manifolds and Jordan $C^{*}$-Algebras, North-Holland Mathematics Studies, 104. Notas de Matemàtica, 96 (North-Holland, Amsterdam, 1985).

[Wa67] L. Waelbroeck, Théorie des Algèbres de Banach et des Algèbres Localement Convexes. Deuxième édition. Séminaire de Mathématiques Supérieures, No. 2 (Été, 1962) (Les Presses de l'Université de Montréal, Montréeal, 1967).

[Wi76] J. Wichmann, 'On the symmetry of matrix algebras', Proc. Amer. Math. Soc. 54 (1976), $237-240$.

DANIEL BELTIŢĂ, Institute of Mathematics, 'Simion Stoilow' of the Romanian Academy, P.O. Box 1-764, RO-014700 Bucharest, Romania e-mail: Daniel.Beltita@imar.ro

KARL-HERMANN NEEB, Department of Mathematics, Darmstadt University of Technology, Schlossgartenstrasse 7, D-64289 Darmstadt, Germany e-mail: neeb@mathematik.tu-darmstadt.de 